\title{
Laser Confocal and Spatially-Resolved Fluorescence Spectroscopy of Porphyrin Distribution on Plasma Deposited Polymer Films
}

\author{
Eduard I. Zenkevich, ${ }^{\mathrm{a}}{ }^{@}$ Joerg Martin, ${ }^{\mathrm{b}}$ Christian von Borczyskowski, ${ }^{\mathrm{b}}$ \\ Tatjana A. Ageeva, ${ }^{\mathrm{C}}$ Valery A. Titov, ${ }^{\mathrm{C}}$ and Valery N. Knyukshto ${ }^{\mathrm{d}}$ \\ ${ }^{a}$ National Technical University of Belarus, Minsk, 220013, Belarus \\ ${ }^{\mathrm{b}}$ Chemnitz University of Technology, Chemnitz, 09107, Germany \\ ${ }^{\mathrm{c}}$ Ivanovo State University of Chemistry and Technology, Ivanovo, 153000, Russia \\ ${ }^{\mathrm{d}}$ B.I. Stepanov Institute of Physics of National Academy of Sciences, Minsk, 220072, Belarus \\ @Corresponding authorE-mail: zenkev@tut.by
}

\begin{abstract}
Laser confocal microscopy and spatially-resolved fluorescence spectroscopy have been used for the study of the interaction of di(p-aminophenyl)etioporphyrin (DAPEP) and tetra(p-aminophenyl)porphyrin (TAPP) molecules with the surface of thin $(7-15 \mu \mathrm{m})$ polypropylene films treated by $0.5 \mathrm{M} \mathrm{KCl}$ solution and activated by air nonequilibrium plasma at the normal atmospheric pressure. Confocal microscopy data (using laser scanning microscope LSM 510, Carl Zeiss) show that after treatment the polymer surface becomes inhomogeneous, and porphyrin moieties are randomly distributed on both film surfaces with a penetration depth of $\sim 1 \mu \mathrm{m}$. On the basis of spatially resolved fluorescence measurements (using a home-built confocal microscope with a time resolution up to 100 ms/frame and high spatial resolution) it has been found that fluorescence spectra of individual spots correspond to monomeric species. It means that spatially closed few porphyrin molecules in the spot are not aggregated and do not interact significantly.
\end{abstract}

\section{Introduction}

At present, synthetic porphyrins and phthalocyanines being fixed on synthetic polymer carries by various methods $^{[1-8]}$ or being polymers themselves ${ }^{[9-12]}$ are the subject of interest both as model compounds of natural polymer-bonded tetrapyrroles (chlorophyll, hemoglobin, cytochrome), ${ }^{[13-19]}$ new biological and catalytically active materials ${ }^{[2-5]}$ as well as modified membranes and sensors. ${ }^{[20-24]}$ The fixation of porphyrins on polymer carrier allows to realize some advantages which are absent when using nonbonded porphyrins. Some of them are as follows: ${ }^{[25]}$ cooperative interactions in polymeric chains, the separation of active centres, the possibility of the specific binding of various substrates on active centres, the increase of tetrapyrrole macrocycle stability, the lowering of the porphyrin toxicity relative to biological environment. In this respect, the evaluation of these combined new porphyrin-polymer properties seems to be perspective both from fundamental and application aspects (biotechnology, biology, pharmacology, medicine and catalysis).

Polymers are a class of materials widely used for a broad field of applications. The covalent and non-covalent attachment of bioactive compounds to functionalized polymer surfaces includes relevant techniques in polymer surface modification such as wet chemical, organosilanization, ionized gas treatments, and UV irradiation. ${ }^{[26]}$ Ion irradiation ranging from several $\mathrm{eVs}$ to $\mathrm{GeVs}$ is a quite efficient tool to modify the properties of polymers like wettability, optical properties, adhesion between organic substrates and polymer surfaces. Ion irradiation is a promising method to generate structures with a modified index of refraction, which is necessary for the guidance of light with different wavelengths in optical devices. ${ }^{[27]}$ Recently reported applications in the areas such as biomedicine, biosensors, enzyme reactors, and textiles, all of which utilize a common set of surface bioconjugation techniques to address these diverse needs, are also discussed in the literature. ${ }^{[26,28]}$

Typically, in order to elucidate the fundamental mechanisms occurring in a revolutionary plasma deposition process, atmospheric pressure plasma deposition as well as to analyze biofunctionalized polymer surfaces, spectral methods (X-ray photoelectron spectroscopy, Fourier transform infrared spectroscopy, atomic force microscopy, quadrupole mass spectrometry and others) as well as nonspectral methods (contact angle, $90^{\circ}$ peel tests, dye assays, biological assays, and zeta potential) are being used. ${ }^{[26,28-30]}$

In this report, we present the results showing the possibilities of the modern experimental methods such as laser confocal microscopy and spatial-resolved fluorescence spectroscopy for the study of the interaction of biologically active molecules, di(p-aminophenyl)etioporphyrin (DAPEP) and tetra( $p$-aminophenyl)porphyrin (TAPP), with the surface of thin $(7.2-15 \mu)$ polypropylene films treated by $0.5 \mathrm{M} \mathrm{KCl}$ solution and activated by air non-equilibrium plasma at normal atmospheric pressure. Specifically, these methods open the possibility to control the distribution of active organic component on the film surface as well as to discriminate the nature (monomeric or aggregated forms) of adsorbed molecular species. The choice of polypropylene films is governed by the fact that this material is one of the most inert polymers with a low surface energy and low sorbtion properties and thus is widely used in medicobiological applications. ${ }^{[26,31]}$ At last, it should be mentioned that the fixation of porphyrins (in monomeric form 
presumably) on polymer film may be considered for possible applications in photodynamic therapy and blood cleaning as well as for the thermostabilization of polymers and the development of optical limiters (using metalloporphyrins and metallophthalocyanines). ${ }^{[25]}$

\section{Experimental}

\section{Sampling}

The synthesis, purification and identification (by absorption, IR and NMR data) of di(p-aminophenyl)etioporphyrin II (DAPEP) molecules were carried out according to, ${ }^{[32]}$ the corresponding procedure for tetra-( $p$-aminophenyl)porphyrin (TAPP) molecules is described in. ${ }^{[33]}$ The structures of the corresponding compounds used for the anchoring to the treated polypropylene films are shown in Figure 1. It was supposedly believed that side active amino groups may be responsible for the non-covalent attachment of porphyrin macrocycles to the polypropylene film surface.

Samples of commercial isotactic polypropylene (PP) films with the thickness of 7-15 $\mu \mathrm{m}$ were cleaned by ethanol and distilled water and dried under vacuum before plasma treatment.

The analysis of ATR-FTIR spectra of untreated but cleaned PP films has shown that the strong own absorption bands are accompanied by the existence of bands belonging to oxygen containing functional groups: i) bands in the range of $3000-3600 \mathrm{~cm}^{-1}$ are characteristic for hydroxyl groups; ii) bands in the region of $1660-1750 \mathrm{~cm}^{-1}$ may be attributed to aldehyde and carboxyl groups; iii) bands at $890 \mathrm{~cm}^{-1}$ (double bonds of vinylidene type) and $1377 \mathrm{~cm}^{-1}$ (branch methyl groups) were also observed. The basic simplified scheme of experimental setup for excitation $\boldsymbol{A}$<smiles></smiles>

of the atmospheric pressure glow discharge (APGD) with liquid electrolyte cathode is shown in Figure 2.

The discharge cell was open to ambient air. DC power supply with the $30 \mathrm{k} \Omega$ ballast resistor was used for the discharge excitation. High voltage (up to $4 \mathrm{kV}$ ) was applied between liquid electrolyte cathode and copper anode placed at the position of $1-5 \mathrm{~mm}$ above the solution surface. The electrolyte solution was connected to the negative pole of the power supply through a $\mathrm{Cu}$ electrode. A discharge current was varied from 10 to $70 \mathrm{~mA}$. A distance between the polymer surface and electrolyte-gas interface was $2 \mathrm{~mm}$, thus both polymer film sides were immersed in the liquid. Typically, samples were of $70 \mathrm{~mm}$ and in length $10 \mathrm{~mm}$ in width. The liquid solution volume was capped of about $70 \mathrm{ml}$. Distilled water and aqueous $\mathrm{KCl}$ solutions of $0.01,0.05,0.1$, and $0.5 \mathrm{M}$ were utilized as a liquid cathode.

Treatment of polymer samples in low-pressure $\mathrm{O}_{2}$ plasma or in afterglow of the $\mathrm{O}_{2}$ plasma was used for surface activation too. The experimental setup for polymer treatment in low-pressure plasma and treatment conditions were described in details earlier. ${ }^{[35]}$

After the treatment, polymer film samples were washed with distilled water and dried under the vacuum at the pressure of $1 \mathrm{~Pa}$. Basically, the main results will be discussed for PP films with attached 10,20-di(p-aminophenyl)ethioporphyrin II (DAPEP) and tetra(p-aminophenyl)porphyrin (TAPP): (1A) is a control sample, non-treated film, $(2 \mathrm{~A})$ is the film treated by $\mathrm{O}_{2^{-}}$ plasma + DAPEP, $(3 \mathrm{~A})$ is the film treated by afterglow of $\mathrm{O}_{2-}^{-}$ plasma + DAPEP, (1B) is the film treated by $\mathrm{O}_{2}$-plasma, (2B) is the film treated by afterglow of $\mathrm{O}_{2}$-plasma, $(3 \mathrm{~B})$ is the film treated in plasma-solution system, $(4 \mathrm{~B})$ is the non-treated film + TAPP, $(5 \mathrm{~B})$ is the non-treated film + DAPEP, $(6 \mathrm{~B})$ is the film treated by afterglow of $\mathrm{O}_{2}$-plasma + DAPP.

$\boldsymbol{B}$

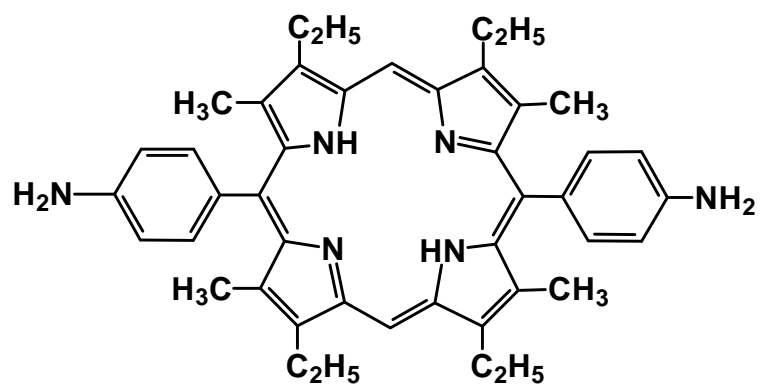

Figure 1. Structures of tetra( $p$-aminophenyl)porphyrin $(A)$ and 10,20-di( $p$-aminophenyl)etioporphyrin II $(B)$.

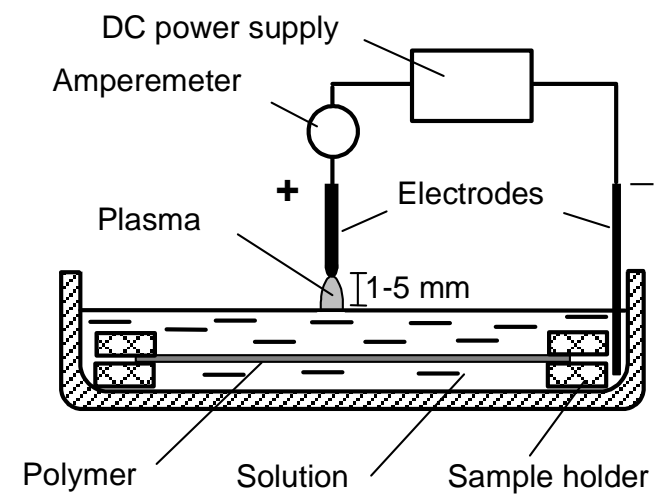

Figure 2. Schematic diagram of the experimental setup for APGD with liquid electrolyte cathode.

\section{Optical and Fluorescence Measurements}

The commercial laser scanning microscope LSM 510 (Release 2.8, Carl Zeiss, Germany, Figure 3) was used in order to measure the thickness of PP films as well to analyze the porphyrin distribution from both sides of the films under study. Technical data of the scanning microscope are as follows: $\mathrm{Z}$ drive scanning stage by DC motor with the optoelectronic coding and the smallest step size of $50 \mathrm{~nm}$; motorized XY scanning stage with the smallest step of $250 \mathrm{~nm}$; the fine focusing stage HRZ 200 with total range of $200 \mu \mathrm{m}$ and the smallest step size $<10 \mathrm{~nm}$; one pinhole per confocal channel; VIS laser modules: $\mathrm{Ar}^{+}$laser 458, 477, 488, 514 $\mathrm{nm}, 30 \mathrm{~mW}$ and HeNe laser $633 \mathrm{~nm}, 5 \mathrm{~mW}$. The spatial resolution of the microscope is not high enough in order to obtain structural changes below $200 \mathrm{~nm}$. In order to measure the thickness of the films, some $x$-z-cuts through the films were recorded in reflection mode, because the resolution is much better than in fluorescence 
regime recording. For each film samples cut images have been obtained with different magnification.

\section{LSM 510 (Carl Zeiss)}

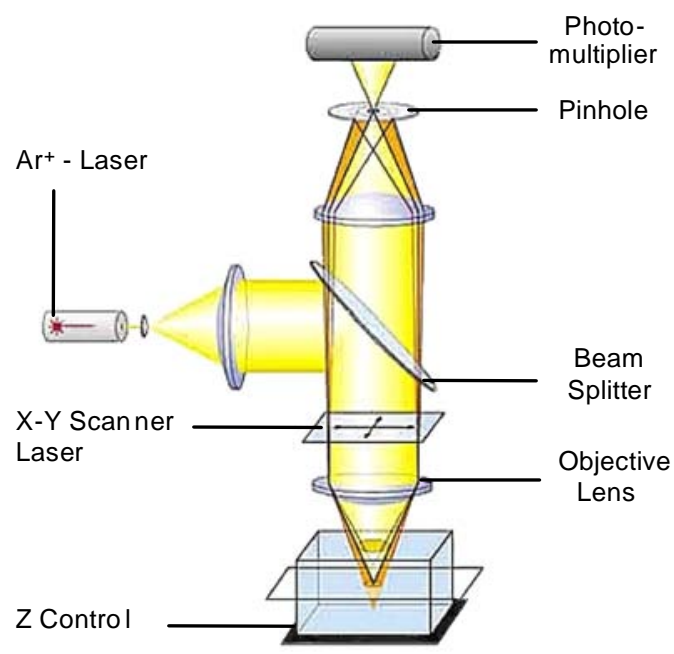

Figure 3. Principal optical scheme of commercial laser scanning microscope LSM 510 (Release 2.8, Carl Zeiss).

Spatially-resolved fluorescence measurements have been carried out by means of a home-built confocal microscope which is shown schematically in Figure $4 .^{[34]}$ The samples were mounted on a closed-loop $x y z$-piezo translation stage (Piezosystems, Karl Zeiss, Jena, Germany) with a maximum scan range of $80 \mu$. The $482 \mathrm{~nm}$ line of a Krypton ${ }^{+}$ion laser or the $514.5 \mathrm{~nm}$ line of an $\mathrm{Argon}^{+}$ion laser were used for the fluorescence excitation of the samples. The light was coupled into the set-up via an optical fiber and focused onto the PP film surface by an objective characterized by 100x magnification and numerical aperture of 0.9 (Carl Zeiss, Jena, Germany). Sample fluorescence was collected by the same objective and imaged onto an avalanche photodiode (APD, EG\&G SPCMAQR-13) by an achromatic lens. By this way spectral images have been visualised on a PC screen. In order to register spatially-resolved fluorescence spectra the second dichroic 50/50-beamsplitter in the detection light path was used for the collecting and imaging the part of the fluorescence emission onto the entrance slit of a grating monochromator (Acton SP-300i) with an attached liquidnitrogen-cooled CCD (Princeton Instruments, USA). This part of the confocal fluorescence microscope is not shown in Figure 4 and presented in details earlier. ${ }^{[34]}$ Longpass Notch filters (Omega Optical, USA) were placed in front of the APD and the spectrometer (530 nm and $505 \mathrm{~nm}$ cut-off wavelengths) to separate the fluorescence signal from the excitation flow. Finally, a home-built confocal microscope is characterized by the following parameters: ${ }^{[34]}$ i) a time resolution up to 100 $\mathrm{ms} /$ frame, ii) a lateral resolution of $235 \mathrm{~nm}$ and a vertical resolution of $970 \mathrm{~nm}$, iii) a detection efficiency of $10 \%$ for the whole confocal set-up (without the second 50/50-beamsplitter).

Commercial spectrophotometers Shimadzu UV 3101PC (Japan) and Carry 500 (USA) were used for absorption measurements, whereas spectrofluorimeter Shimadzu RF5101PC (Japan) and a high sensitive home-made recording complex both calibrated for the spectral response of the detection channel were used for usual steady-state fluorescence measurements. The details of experimental technique have been reported earlier. ${ }^{[36,37]}$

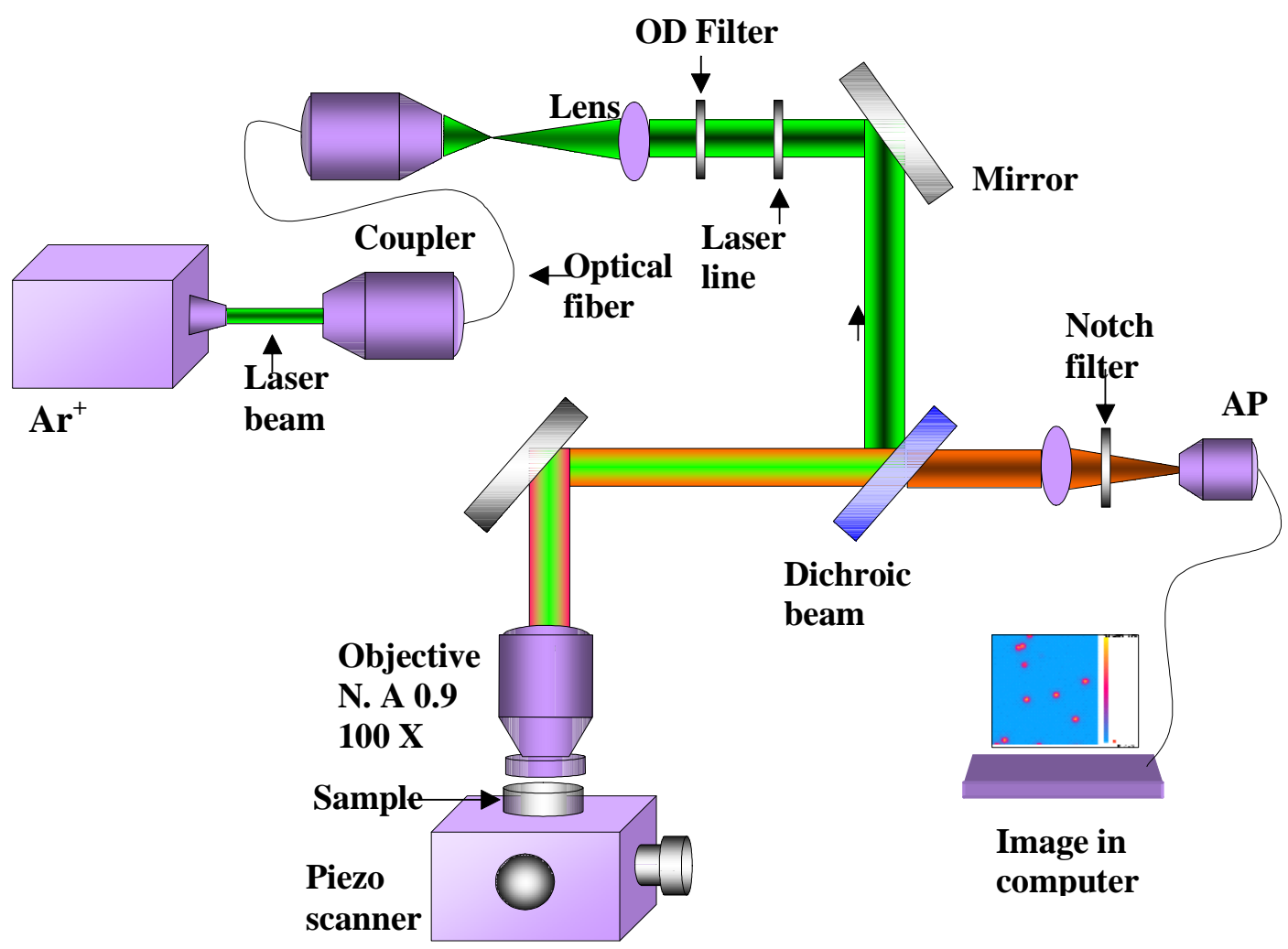

Figure 4. Principal optical scheme of home-built confocal microscope for fluorescence imaging and spectra detection. 

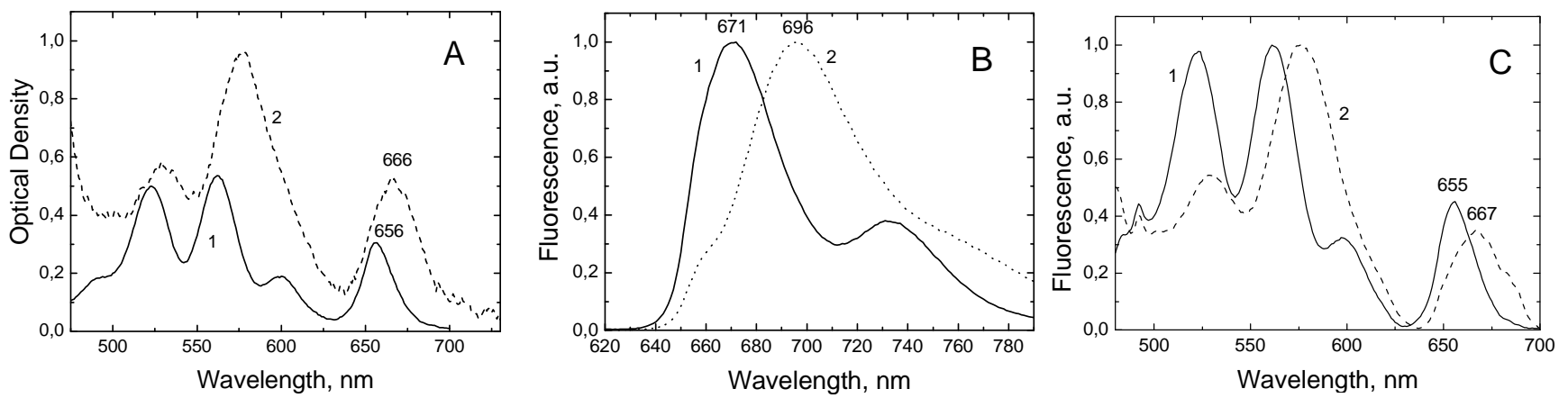

Figure 5. Absorption (A), fluorescence $\left(B, \quad \lambda_{\text {exc }}=420 \mathrm{~nm}\right)$ and fluorescence excitation $\left(C, \quad \lambda_{\text {reg }}=720 \mathrm{~nm}\right)$ spectra of tetra(p-aminophenyl)porphyrin (TAPP) in toluene (1) and pyridine (2) at $295 \mathrm{~K}$.

\section{Results and Discussions}

\section{Usual Steady-State Fluorescence Spectra}

It should be mentioned that spectral properties of DAPEP and TAPP molecules in solutions depend significantly on the solvent properties. As it is presented in Figure 5 for TAPP, when transition from non-polar toluene to pyridine the noticeable transformation of absorption spectra is observed in the visible region: a significant broadening of all bands accompanied by the shift to the red region by $\sim 12 \mathrm{~nm}$, the four-band spectrum transforms to the three-band one. Fluorescence spectra in pyridine are also broadened by $\sim 2$ times with respect to those registered in toluene. Stokes shift between $Q(0,0)$-bands in absorption and fluorescence is essentially higher in pyridine and amounts to $600 \mathrm{~cm}^{-1}$ in comparison with that of $360 \mathrm{~cm}^{-1}$ in toluene. Nevertheless, fluorescence excitation spectra coincide to absorption spectra both in

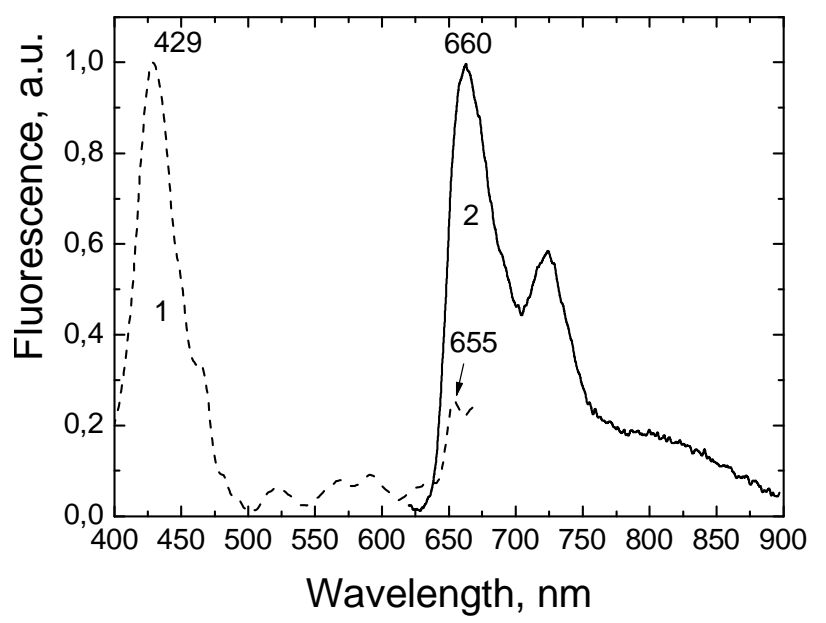

Figure 6. Fluorescence excitation $\left(1, \quad \lambda_{\text {reg }}=720 \mathrm{~nm}\right)$ and fluorescence $\left(2, \quad \lambda_{\text {exc }}=420 \mathrm{~nm}\right) \quad$ spectra of tetra $(p$-aminophenyl)porphyrin (TAPP) attached to polypropylene film 6B, treated in afterglow of $\mathrm{O}_{2}$-plasma $(295 \mathrm{~K})$. toluene and pyridine thus confirming monomeric state of TAPP molecules in both solvents.

The observed experimental spectral findings may be connected with two reasons at least: the reorganisation of solvate shell and the conformational dynamics of tetrapyrrole macrocycle for DAPEP and TAPP molecules in the excited $\mathrm{S}_{1}$-state due to the interaction of meso-phenyl rings with bulky side $\beta$-substituents in pyrrole rings (especially in the case of DAPEP ${ }^{[38,39]}$ ).

Figure 6 shows standard fluorescence and fluorescence excitation spectra registered for PP film $6 \mathrm{~B}$, treated by $\mathrm{O}_{2}$-plasma in after-emission zone and then activated by TAPP (it was difficult to record appropriate absorption spectra because of a small content of porphyrin molecules on the PP film, interference effects and scattering). Evidently, both spectra belong to TAPP molecules. Noteworthy, for TAPP on the PP film, the position of $Q(0,0)$-band in fluorescence excitation spectrum is the practically same as that being observed in toluene, while the fluorescence maximum is measured to be at 660 $\mathrm{nm}$, that is relatively shorter in comparison with $\lambda_{\max }=671 \mathrm{~nm}$ in non-polar toluene. The decrease of stokes shift down to $\sim 70 \mathrm{~cm}^{-1}$ in comparison with $360 \mathrm{~cm}^{-1}$ or $600 \mathrm{~cm}^{-1}$ in solutions may be caused by a more rigid fixation of TAPP (and DAPEP) molecules on the PP film surface. At last, the fluorescence of a control sample 1A (non-treated film without porphyrins) is very low and characterized by admixture of luminescent dirt.

\section{Confocal Microscopy of Polypropylene Films Without and with Porphyrins}

The basic results obtained by confocal microscopy for PP films were obtained for more than 70 images. Here, we present some statistical results characterizing the possibilities of this method for the analysis of the film thickness and porphyrin distribution on both sides of the film. In order to measure the thickness of the films, $x-z-$ cuts through the films were recorded in reflection mode (excitation by $514.5 \mathrm{~nm}$ line of the argon ion laser), because the resolution is much better than in fluorescence regime recording. 


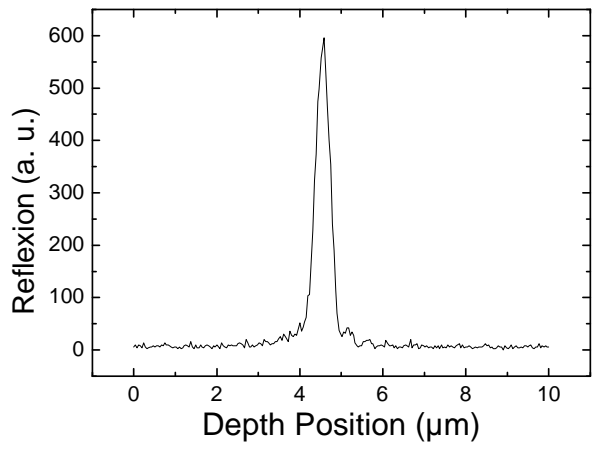

$\boldsymbol{A}$

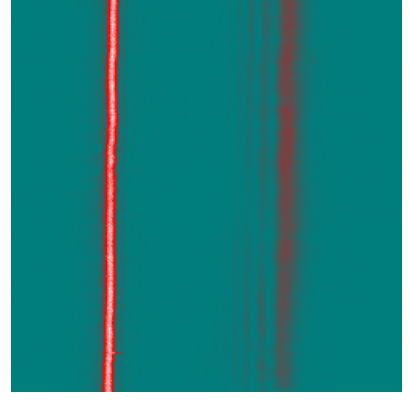

$\boldsymbol{B}$

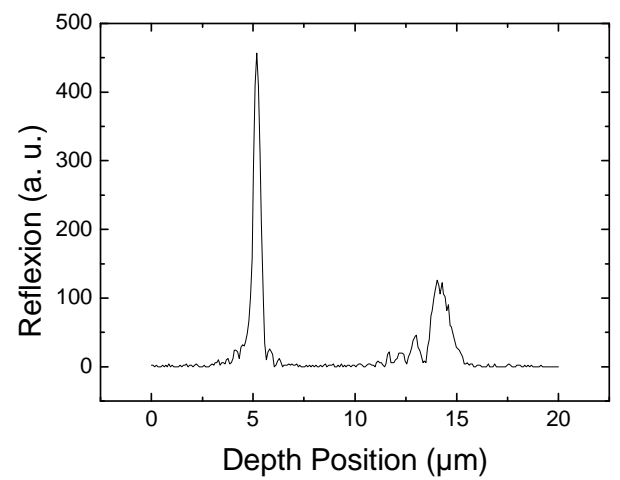

C

Figure 7. Depth profile (A), cut of the reflection image with a size of $20 \times 20 \mu \mathrm{m}^{2}$ (B) and depth profile (C) through the film A (without porphyrin).

For each film samples cut images were obtained with different magnification. Figure 7A shows the depth resolution which was recorded with a mirror and is estimated by $\sim 700 \mathrm{~nm}$. The analysis of the film images $(x$-z-cut, Figure 7) shows two peaks: the left peak originates from the upper side of the film, the right corresponds to the lower one. The oscillations in the peak intensities are due to interference in the film. From these kinds of images film thicknesses were extracted using the fitting procedure by two Gaussian components and displayed in Table 1.

Table 1. Film thicknesses obtained in reflection mode.

\begin{tabular}{lccccccccc}
\hline Films & $1 \mathrm{~A}$ & $1 \mathrm{~B}$ & $2 \mathrm{~A}$ & $2 \mathrm{~B}$ & $3 \mathrm{~A}$ & $3 \mathrm{~B}$ & $4 \mathrm{~B}$ & $5 \mathrm{~B}$ & $6 \mathrm{~B}$ \\
\hline $\begin{array}{l}\text { Thick- } \\
\text { ness } \\
{[\mu \mathrm{m}]}\end{array}$ & 9.03 & 8.75 & 9.00 & 8.76 & 9.18 & 8.82 & 9.02 & 8.96 & 9.03 \\
\hline
\end{tabular}

Description of the films with and without porphyrins is presented in the Experimental part.

It should be mentioned that even though the depth resolution of the setup is only $700 \mathrm{~nm}$, the position of the peaks can be quantified with a much higher accuracy. This is only depends on the signal-to-noise ratio, in our case the experimental depth resolution is estimated to be $\sim 50 \mathrm{~nm}$. The corresponding profiles obtained in the fluorescence mode (especially for the lower side of the film) are wider thus giving less accuracy in the film thickness measurements. At last, from it follows the above measurements that the detected deviations in the film thicknesses are due to the manufacturing process presumably.

After recording cut images lateral images (in reflection and fluorescence modes) of PP film surfaces were measured and visualized. For all the films, the obtained pictures look quite similar, that means one could not detect pronounced effects of the plasma treatment (Figure 8). In some respect, the reason of that may be connected with a relatively low resolution of the microscope which is not high enough in order to analyze structural changes below $200 \mathrm{~nm}$. Dark areas in the upper left and lower right corners of the reflection image are due to the high depth resolution and the ripples of the film. So, the more informative part of the film is a small bright central area. So, if one could manage to put the films uniformly on a substrate the better images may be obtained. Additionally there is much dirt (black spots) on the films remaining after the manufacturing process, which reduces the quality of the images.

Now we would like to come to the fluorescence part of the work which seems to be more interesting.
$\boldsymbol{A}$

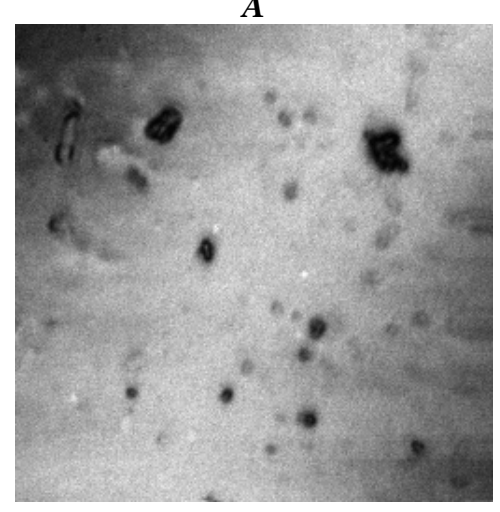

$\boldsymbol{B}$

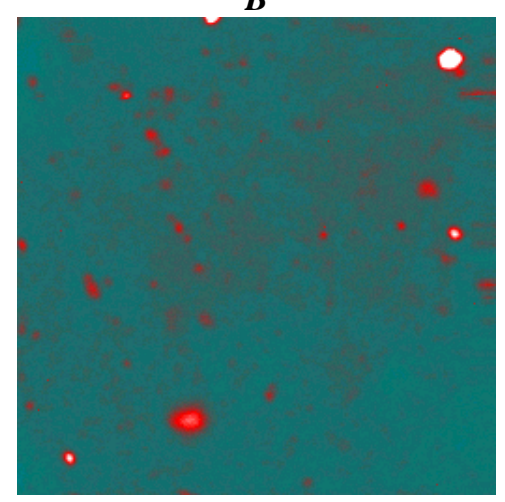

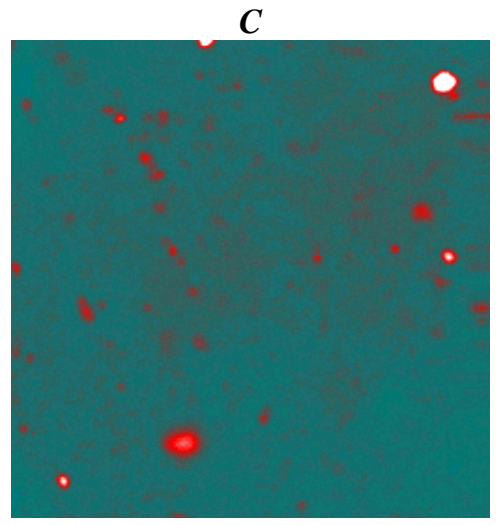

Figure 8. Reflexion lateral image of a control non-treated PP film 1A (A) as well as fluorescence lateral images of films $2 \mathrm{~A}$ (B, film treated by $\mathrm{O}_{2}$-plasma in zone of positive range of charge $+\operatorname{di}(p$-aminophenyl)etioporphyrin II, DAPEP) and $6 \mathrm{~B}(\mathrm{C}$, film treated in afterglow of $\mathrm{O}_{2}$-plasma + tetra( $p$-aminophenyl)porphyrin, TAPP). All images have a size of $20 \times 20 \mu \mathrm{m}^{2}, \lambda_{\text {exc }}=514.5 \mathrm{~nm} \mathrm{by} \mathrm{Ar}^{+}$laser, $\lambda_{\text {det }}>530 \mathrm{~nm}$ at fluorescence mode. 


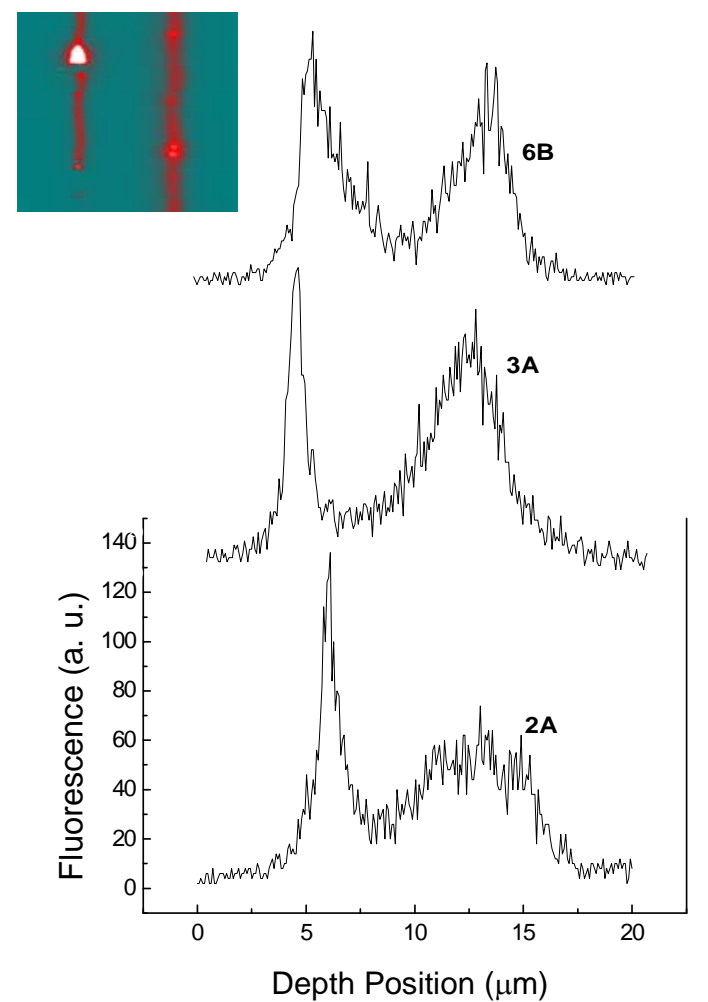

Figure 9. Depth profiles of the fluorescence image cuts with a PP films size of $20 \times 20 \mu \mathrm{m}^{2}: 2 \mathrm{~A}$ - film treated by $\mathrm{O}_{2}$-plasma in zone of positive range of charge $+\mathrm{di}(p$-aminophenyl)etioporphyrin II, DAPEP); $3 \mathrm{~A}$ - is the film treated by $\mathrm{O}_{2}$-plasma in after-emission zone + DAPEP and 6B - film treated in afterglow of $\mathrm{O}_{2}$-plasma one + tetra $\left(p\right.$-aminophenyl)porphyrin, TAPP. $\lambda_{\text {exc }}=514.5 \mathrm{~nm}$ by $\mathrm{Ar}^{+}$laser, $\lambda_{\operatorname{det}}>530 \mathrm{~nm}$ at fluorescence mode. The inset shows the cut of the fluorescence image with a size of $20 \times 20 \mu \mathrm{m}^{2}$ for PP film $3 \mathrm{~A}$.

The necessary measurements were carried out in the same way as for the reflexion mode. The excitation conditions $\left(\lambda_{\text {exc }}=514.5 \mathrm{~nm}\right.$ by $\mathrm{Ar}^{+}$laser $)$were suitable for the direct excitation of porphyrin molecules thus providing enough signal for $x$-z-cuts recording. Some of the results are shown in Figure 9. Like in reflexion mode, the left peak corresponds to the upper side (which is also the reaction side in case of plasma treatment), the right one corresponds to the lower side. The analysis of fluorescence profiles of cut images shows rather strong differences in the distributions of the porphyrins on film surfaces treated by various ways. From these findings it may be concluded that after plasma treatment the polymer surface becomes nonhomogeneous, and porphyrin moieties are seen to be randomly distributed on both film surfaces with a penetration depth of $\sim 1 \mu \mathrm{m}$.

\section{Spatially-Resolved Fluorescence Spectroscopy of Polypropylene Films with and without Porphyrins}

One primary motivation for the studying the spatiallyresolved emission of individual objects (more clearly, individual organic molecules, ${ }^{[40]}$ porphyrins, ${ }^{[41,42]}$ spins $^{[43]}$ or semiconductor quantum $\operatorname{dots}^{[44]}$ ) in solids, liquids, proteins or on interfaces and surfaces is due to the possibilities to elucidate both the local environment influence (heterogeneous by nature) and the individual object state and properties, so that the behaviour of individuals may not be fully represented by the standard (large number $\mathrm{N}$ ) ensemble average. At present, conventional confocal microscope technique (home-built presumably) permits to carry out steady-state and kinetic fluorescent measurements in a wide temperature range including liquid helium temperatures. The development of high-spatial-resolution optical microscopy has fostered a considerable progress in this field. Briefly, this method has now broad interdisciplinary impact, ranging from physical and analytical chemistry to biophysics, medicine, material science and nanotechnology.

Our use of the spatially-resolved fluorescence spectroscopy for polypropylene films activated by porphyrins is to gather information about the spatial arrangement of these molecules adsorbed on film surfaces as well as to clearly recognize the state of the molecules on the PP surface (monomeric or aggregated, at least). As it is shown in Figure 5, fluorescence spectra of TAPP molecules in both toluene and pyridine are relatively wide being ensemble averaged due to inhomogeneous broadening. For TAPP on the PP film, the fluorescence band (detected from the square of $0.5 \mathrm{~cm}^{2}$ ) is noticeably narrower with respect to toluene solution and its maximum is measured to be at $660 \mathrm{~nm}$, that is relatively shorter in comparison with $\lambda_{\max }=671 \mathrm{~nm}$ in non-polar toluene (Figure 6). The decrease of stokes shift down to $\sim 70 \mathrm{~cm}^{-1}$ in comparison with 360 $\mathrm{cm}^{-1}$ or $600 \mathrm{~cm}^{-1}$ in solutions is proposed to be caused by a more rigid fixation of TAPP molecules on the PP film surface. In this case, the standard steady-state registration gives us TAPP emission which is also ensemble averaged due to inhomogeneous broadening caused by the specific interaction of every TAPP molecule with PP film surface.

In the first step of data evaluation, the fluorescence images of PP films without and with porphyrin molecules have been analyzed (Figure 10, left side A, B, C). Figure $10 \mathrm{~A}$ shows that even for pure film without porphyrin there are some fluorescing admixtures visualized (in fluorescence mode) like relatively bright spots remaining after the industry manufacturing process and non-eliminated by the solvent treatment.

It is seen from Figure $10 \mathrm{~B}$ that for the film treated by plasma and then activated by TAPP, the number of fluorescing spots becomes relatively larger. We attribute it to the appearance of porphyrin molecules on PP film surface. In order to verify such a possibility we have excited these bright spots by the narrow laser beam and detected the spectral distribution of this emission using home-built confocal microscope. In Figure $10 \mathrm{~B}$ one examined spot is shown by a white arrow. For the comparison, the other arrow in Figure $10 \mathrm{~B}$ shows the empty place on PP film surface, emission from which was used for the background emission detection at the same experimental conditions. We want to emphasize that spatially-resolved fluorescence measurements need a relatively high laser power densities thus leading to a noticeable photobleaching of organic molecules. Really, Figure $10 \mathrm{C}$ shows that after spectral detection the brightness of the investigated spot became smaller.

On the right side of Figure 10 the background emission of the film (D), total fluorescence spectrum (E) and the spectrum with subtracted film emission (F) are presented being obtained for the empty film place and the 
spot shown by arrows on left images. The comparison of fluorescence profiles and the maximum position obtained for the spots (Figure $10 \mathrm{~F}$ ) with those detected in ensemble measurements (Figure 6) shows a good coincidence.

Thus, for bright spots the emission originates from TAPP molecules, and our assumption about the nature of the majority of bright spots seems to be justified. The same results were obtained in numerous experiments with both TAPP and DAPEP molecules absorbed on PP films treated in various plasma conditions.

At last, it should be mentioned that the lateral resolution of the confocal setup does not exceed $\Delta \mathrm{l} \approx 235 \mathrm{~nm}$ and a vertical resolution is of $970 \mathrm{~nm}$. This means that spots may contain few or even more porphyrin molecules disposed closely to each other. Correspondingly, the possibility of porphyrin aggregation effects should be taken into account. A set of experimental data which we have at the moment leads to the following conclusions. From one hand, as it was mentioned above, it was difficult to record appropriate absorption spectra of the samples because of a small content of porphyrin molecules on the PP film, interference effects and scattering. From the other hand, a spontaneous aggregation of porphyrins in solutions does not manifest significantly in absorption spectra (small spectral shift and bands broadening, the more sensitive is Soret band, see a review chapter ${ }^{[12]}$ ). For TAPP molecules on PP film surfaces, ensemble average fluorescence excitation spectra in UV-vis region (relative intensities of the bands, Figure 6) coincide with the same distribution measured for monomeric TAPP molecules in toluene (insert in Figure 6). Noteworthy also is the full coincidence in the positions of Soret bands $(429 \mathrm{~nm})$ in both spectra. It is seen also that the intensities ratio of vibronic $I(1,0)$ to pure electronic $I(0,0)$ bands in fluorescence spectra of TAPP is
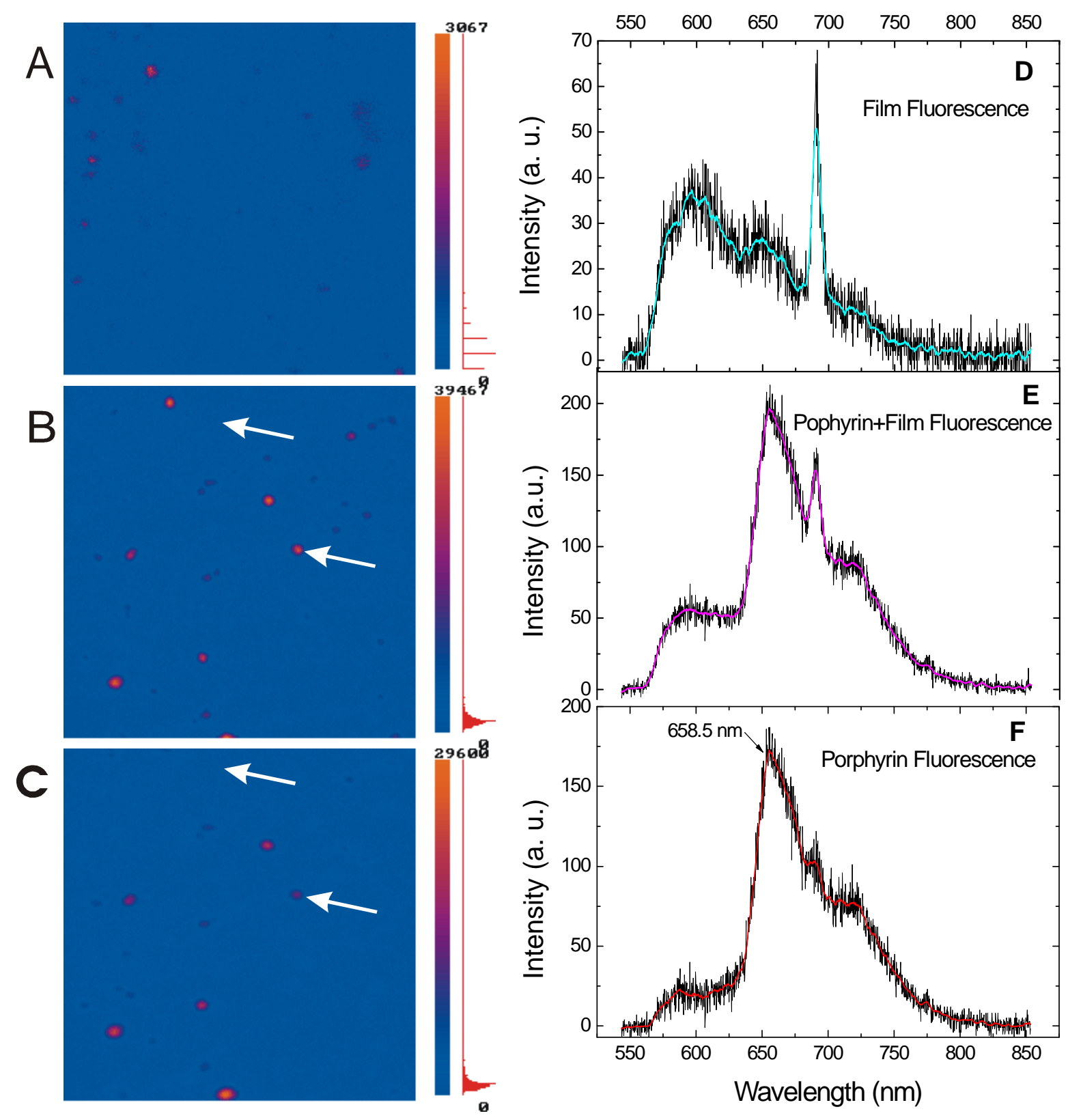

Figure 10. Fluorescence images of pure PP film with a size of $20 \times 20 \mu \mathrm{m}^{2}$, upper side (A) and film treated in afterglow of $\mathrm{O}_{2}$-plasma + tetra( $p$-aminophenyl)porphyrin, TAPP, before (B) and after (C) laser spectral measurements. Fluorescence spectra of the PP film spot without porphyrin (D) and with porphyrin (E); the spectrum shown in part (F) represents fluorescence of the attached porphyrin molecules with subtracted admixture of the PP film emission. $\lambda_{\mathrm{exc}}=407 \mathrm{~nm}$, power density $\mathrm{N}=25 \mu \mathrm{W} / \mu \mathrm{m}^{2}, 295 \mathrm{~K}$. 
practically the same for toluene solutions of monomeric porphyrin (Figure 5, spectrum 1), ensemble average fluorescence of PP surface (Figure 6, spectrum 2) and fluorescence spectrum of the PP film spot with porphyrin (Figure $10 \mathrm{~F}$ ), and is equal to $I(1,0) / I(0,0) \approx 0.4 \div 0.5$. The decrease of stokes shift down to $\sim 70 \mathrm{~cm}^{-1}$ in comparison with $360 \mathrm{~cm}^{-1}$ in toluene solution may be caused by a more rigid fixation of TAPP molecules on the PP film surface. Correspondingly, in the spot the fluorescing species of TAPP molecules as monomeric ones may be taken as proved.

In this respect, some additional arguments should be taken into account. Typically, spontaneous porphyrin aggregates and oligomeric/polymeric porphyrins are characterized by low fluorescence efficiencies. ${ }^{[10-12]}$ Accordingly, TAPP spontaneous aggregates are hardly detected by emission with respect to monomers in one spot. But in the case of the considerable existence of aggregated TAPP species together with monomeric form in one spot at relatively small distances, the non-radiative resonant energy transfer "monomer $\rightarrow$ aggregate" should take place thus leading to the strong quenching of the monomer fluorescence (fluorescence efficiency decrease and decay shortening). Nevertheless, our preliminary time-correlated single photon counting measurements have shown that fluorescence decay of TAPP in spots (three have been analysed) is of $8.7 \div 9.3 \mathrm{~ns}$. These values are typical for monomeric porphyrins of various types in liquid solvents in the presence of dissolved oxygen. ${ }^{[12,13,36,38]}$ This situation may be explained by two ways: i) TAPP molecules are in monomeric form presumably in the spot; ii) there is a distribution of monomers and aggregates in the spot, but intercenter monomer-aggregate distances are relatively large and the effective energy transfer "monomer $\rightarrow$ aggregate" does not realise. The statistical analysis of time-correlated single photon counting measurements being obtained for various spots and concentrations of TAPP and DAPEP molecules on PP surface is under the way.

\section{Conclusions}

We have studied the interaction of $\operatorname{di}(p$-aminophenyl)etioporphyrin (DAPEP) and tetra( $p$-aminophenyl)porphyrin (TAPP) molecules with the surface of thin $(7-15 \mu \mathrm{m})$ polypropylene films using laser confocal microscopy and spatially-resolved fluorescence measurements. On the basis of these contemporary spectroscopic methods in the combination with ensemble averaged measurements it has been elucidated that fluorescence spectra of individual bright spots on film surfaces correspond to porphyrin monomeric species. Thus it seems to be concluded that spatially closed few porphyrin molecules in the spot do not form aggregates. Polypropylene films with low surface energy and low sorbtion properties being surfacely activated by porphyrins (in monomeric form known as affective sensitizers of singlet oxygen ${ }^{1} \Delta_{\mathrm{g}}{ }^{[45,46]}$ ) may be used in photodynamic therapy and blood cleaning. Porphyrins fixed on polymer surfaces are considered for possible applications and thermostabilization of polymers (especially metallo-porphyrins). ${ }^{[25]}$ Samples "porphyrinpolymer", like tetrapyrrole compounds forming strongly absorbing excited states in solutions, ${ }^{[47,48]}$ may be used as optical limiters, which can protect sensors or human eyes from optical damage. Concerning our interests, two practical questions (blood cleaning and optical limiters) based on these systems will be the subject of further thorough investigations.

Acknowledgements. This work was supported by BRFFR Grant No Ф07MC-022 (Belarus, Germany) and GKPNI «KMS-17» (Belarus). Authors thank also Prof. A. Semeikin and Dr. S. Syrbu for the synthesis and purification of DAPEP and TAPP molecules.

\section{References}

1. Shtilman M.I. Immobilization on polymers. Utrecht, Tokio, VSP, 1991.

2. Pomogailo D.A. Uflyand I.E. Macromolecular Metallochelates Moscow, Khimiya 1991. 304.

3. Polymeric Reagents and Catalysts (Ford U.E., Ed.) Moscow, Khimiya 1991. 256 p.

4. Solov'eva A.B., Timashev S.F. Uspekhi Khimii 2003, 72, 1081-1097.

5. Lisichkin G.F., Yuffa A.Ya. Heterogeneous Metalocomplex Catalysts. Moscow, Khimiya 1981, 160

6. Valkova L., Borovkov N., Koifman O., Kutepov A., Berzina T., Fontana M., Rella R., Valli L. Biosensors and Bioelectronics 2004, 20, 1177-1182.

7. Wöhrle D. In Phthalocyanines: Properties and Applications. Vol. 1. (Leznoff C.C., Lever A.B.P., Eds.) N.Y, VCH, 1989, 55-87.

8. Ulbricht M. Polymer 2006, 47, 2217-2262.

9. Kosal M.T., Suslick K.S. J. Solid State Chem. 2000, 152, 8798.

10. Nakai K., Kurotobi K., Osuka A., Uchiyama M., Kobayashi N. J. Inorg. Biochem. 2008, 102, 466-471.

11. Yang J., Park M., Yoon Z., Hori T., Peng X., Aratani N., Dedecker P., Hotta J., Uji-I H., Sliwa M., Hofkens J., Osuka A., Kim D. J. Am. Chem. Soc. 2008, 130, 1879-1884.

12. Zenkevich E.I., von Borczyskowski C. In Handbook of Polyelectrolytes and Their Applications Vol. 2 (Tripathy S.K., Kumar J., Nalwa H.S., Eds.) USA: American Scientific Publishers, 2000, Ch. 11, 301-348.

13. Zenkevich E.I., von Borczyskowski C., Shulga A.M., Bachilo S.M., Rempel U., Willert A. Chemical Physics 2002, 275, 185-209.

14. Chambron J.-C., Heitz V., Sauvage J.-P. In The Porphyrin Handbook Vol. 6 (Kadish K.M., Smith K.M., Guilard R., Eds) New York, Academic Press, 2000, Ch.40, 1-35.

15. Sessler J.L., Wang B., Springs S.L., Brown C.T. In Comprehensive Supramolecular Chemistry Vol. 4 (Marakami Y., Ed.) Oxford, 1996, 311.

16. Ogoshi H., Mizutani T., Hayashi T., Kuroda Y. In The Porphyrin Handbook Vol. 7 (Kadish K.M., Smith K.M., Guilard R., Eds) New York, Academic Press, 2000, Ch. 46, 279.

17. Gust D., Moore T.A. In The Porphyrin Handbook Vol. 8 (Kadish K.M., Smith K.M., Guilard R., Eds) New York, Academic Press, 2000, Ch. 57, 57.

18. Fukuzumi S. In The Porphyrin Handbook Vol. 8 (Kadish K.M., Smith K.M., Guilard R., Eds) New York, Academic Press, 2000, 115.

19. Hayes R.T., Walsh C.J., Wasielewski M.R. J. Phys. Chem. A. 2004, 108, 2375-2383.

20. DiMarco G., Lanza M. Sensors and Actuators B. 2000, 63, 42.

21. Amao Y., Tabuchi Y., Yamashita Y., Kimura K. Eur. Polymer J. 2002, 38, 675 .

22. Nakagawa K., Kumon K., Tsutsumi C., Tabuchi K., Kitagawa T., Sadaoka Y. Sensors and Actuators B, 2000, 65, 138. 
23. Tsivadze A.Yu. Uspekhi Khimii 2004, 73, 6.

24. Tsivadze A.Yu. Crown-compounds as ,ion traps“, in Encyclopedia «State-of-the-art natural science» Vol. 6 Moscow, Magistr-Press, 2000, 247.

25. Koifman O.I., Ageeva T.A. Porphyrinopolymers Moscow: Phys.-Math. Lit. Press, 2006194

26. Goddard J.M., Hotchkiss J.H. Progress in Polymer Science 2007, 32, 698-725.

27. Rück D.M. Nuclear Instruments and Methods in Physics Research Section B: Beam Interactions with Materials and Atoms 2000, 166-167, 602-609.

28. Zaporojtchenko V., Zekonyte J., Faupel F. Nuclear Instruments and Methods in Physics Research Section B: Beam Interactions with Materials and Atoms 2007, 265, 139145.

29. Nasef M.M., Saidi H., Dahlan K.Z.M. Polymer Degradation and Stability 2002, 75, 85-92.

30. Rietveld I.B., Kobayashi K., Yamada H., Matsushige K. Journal of Colloid and Interface Science 2006, 298, 639-651.

31. Golubchikov O.A., Ageeva T.A., Titov V.A., Vershinina I.A., Shikova T.G., Semeikin A.S., Maksimov A.I., Zenkevich E.I. Method of the surface modification for propylene material. The Patent of Russian Federation № 2223982, Invention Bulletin №5 (20.02.2004).

32. Syrbu S.A., Glazunov A.V., Semeikin A.S. Izv. Vysch. Uchebn. Zaved. Khim. Teknol. 2006, 49, 122-123.

33. Semeikin A.S., Koifman O.I., Berezin B.D. Izv. VUZov Khim. Teknol. 1985, 28, 47-51.

34. Cichos F., Martin J., von Borczyskowski C. Phys. Rev. B 2004, 70, 115314.

35. Choi H.S., Rybkin V.V, Titov V.A., Shikova T.G., Ageeva T.A. Surface and Coatings Technology 2006, 4479-4488.
36. Zenkevich E., Sagun E, Knyukshto V., Shulga A., Mironov A., Efremova O., Bonnett R., Pinda Songca S., Kassem M. J. Phochem. Photobiol. B: Biol. 1996, 33, 171-180.

37. Zenkevich E., Cichos F., Shulga A., Petrov E., Blaudeck T., von Borczyskowski C. J. Phys. Chem. B. 2005, 109, 86798692.

38. Knyukshto V., Zenkevich E., Sagun E., Shulga A., Bachilo S. Chem. Phys. Lett. 1999, 304, 97-108.

39. Avilov I.V., Zenkevich E.I., Sagun E.I., Filatov I.V. J. Phys. Chem. A. 2004, 108, 5684-5691.

40. Hinze G., Haase M., Nolde F., Muellen K., Basche Th. J. Phys. Chem. A. 2005, 109, 6725-6729.

41. Starukhin A., Shulga A., Sepiol J., Kolos R., Renn A., Wild U.P. Single Molecules 2001, 2, 203 - 206.

42. Bricas R.P., Troxler T., Hochstrasser R.M., Vinogradov S.A. J. Am. Chem. Soc. 2005, 127, 11851-11862.

43. von Borczyskowski C., Koehler J., Moerner W.E., Orrit M., Wrachtrup J. Appl. Magn. Reson. 2007, 31, 665-676.

44. Shimizu K.T., Woo W.K., Fisher B.R., Eisler H.J., Bawendi M.G. Phys. Rev. Lett. 2002, 89, 117401.

45. Dougherty T.J., Gomer C.J., Henderson B.W., Jori G., Kessel D., Kovbelik M., Moan J., Peng Q. J. Natl. Cancer Inst. 1998, 90, 889-905.

46. Grin, M.A., Lonin I.S., Fediounin S.V., Tsiprovskiy A.G., Strizhakov A.A., Tsygankov A.A., Krasnovsky A.A., Mironov A.F. Mendeleev Commun. 2007, 17, 209-211.

47. Perry J.W., Mansour K., Lee I.-Y.S., Wu X.-L., Bedworth P.V., Chen C.-T., Ng D., Marder S.R., Miles P., Wada T., Tian M., Sasabe H. Science 1996, 273, 1533 - 1536.

48. Kuznetsova R., Kopylova T., Mayer G., Svetlichnyi V., Samsonova L., Tel'minov E., Sergeev A. Proc. SPIE Int. Soc. Opt. Eng. 1997, 3403, 186-191. 